\title{
A Coordinated Radio Afterglow Program
}

\author{
D. A. Frail ${ }^{1}$, S. R. Kulkarni ${ }^{2}$, M. H. Wieringa ${ }^{3}$, G. B. Taylor ${ }^{1}$, \\ G. H. Moriarty-Schieven ${ }^{4}$, D. S. Shepherd ${ }^{1}$, R. M. Wark ${ }^{3}$, \\ R. Subrahmanyan ${ }^{3}$, D. McConnell ${ }^{3}$, and S. J. Cunningham ${ }^{3}$ \\ ${ }^{1}$ National Radio Astronomy Observatory, Socorro, NM $87801^{1}$ \\ ${ }^{2}$ Division of Physics, Mathematics, and Astronomy 105-24, Caltech, Pasadena, CA 91125 \\ ${ }^{3}$ Australia Telescope National Facility, CSIRO, Epping 2121, Australia \\ ${ }^{4}$ Joint Astronomy Centre, 600 A'Ohoku Place, Hilo, HI 96720
}

\begin{abstract}
We describe a ground-based effort to find and study afterglows at centimeter and millimeter wavelengths. We have observed all well-localized gamma-ray bursts in the Northern and Southern sky since BeppoSAX first started providing rapid positions in early 1997. Of the 23 GRBs for which X-ray afterglows have been detected, 10 have optical afterglows and 9 have radio afterglows. A growing number of GRBs have both $\mathrm{X}$-ray and radio afterglows but lack a corresponding optical afterglow.
\end{abstract}

\section{INTRODUCTION}

BeppoSAX revolutionized gamma-ray burst (GRB) astronomy not only through its discovery of $\mathrm{X}$-ray afterglows but also through the dissemination of accuratc and timely GRB positions to ground-based observers, who then conduct searches for afterglows at optical and radio wavelengths. Our collaboration uses the interferometer facilities of the Very Large Array (VLA), the Australia Telescope Compact Array (ATCA), the Very Long Baseline Array (VLBA) and the Owens Valley Radio Observatory (OVRO) Interferometer. At high frequencies, we use single dish telescopes which include the James Clerk Maxwell Telescope (JCMT) and the OVRO 40-m Telescope. All afterglow searches begin with the VLA in the northern hemisphere $\left(\delta>-45^{\circ}, \sigma_{\mathrm{rms}}=45 \mu \mathrm{Jy}\right.$ in $\left.10 \mathrm{~min}, \mathrm{FOV} \simeq 5^{\prime}\right)$ and the ATCA in the southern hemisphere $\left(\delta<-45^{\circ}, \sigma_{\mathrm{rms}}=45 \mu \mathrm{Jy}\right.$ in $240 \mathrm{~min}$, FOV $\left.\simeq 5^{\prime}\right)$, typically at a frequency of $8.5 \mathrm{GHz}$, which provides a balance between sensitivity and fieldof-view. Follow-up programs at the other radio facilities are begun after a VLA or ATCA transient is discovered.

As with quasars, radio observations provide unique diagnostics complementary to those obtained at X-ray and optical wavelengths. Our collaboration has discovered

1) The NRAO is a facility of the National Science Foundation, operated under cooperative agreement by Associated Universities, Inc.

CP526, Gamma-Ray Bursts: $5^{\text {th }}$ Huntsville Symposium, edited by R. M. Kippen, et al. (C) 2000 American Institute of Physics 1-56396-947-5/00/\$17.00 


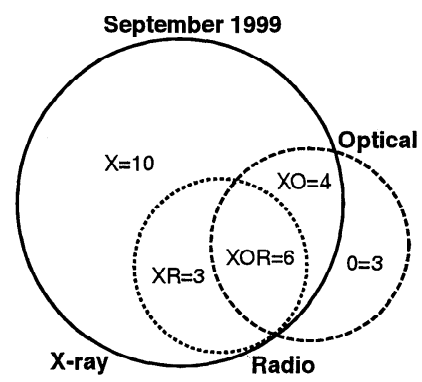

FIGURE 1. A Venn diagram showing the detection statistics for 26 well-localized GRBs. Of the $23 \mathrm{GRBs}$ for which X-ray afterglows have been detected to date, 10 have optical afterglows $(\mathrm{XO}+\mathrm{XOR})$ and 9 have radio afterglows $(\mathrm{XR}+\mathrm{XOR})$. In total there are 13 optical and/or radio afterglows with corresponding $\mathrm{X}$-ray afterglows (XO $+\mathrm{XR}+\mathrm{XOR}$ ). Only 6 GR.Bs have afterglows detected in all three bands (XOR).

all known radio afterglows to date, leading to a number of important results: the direct demonstration of relativistic expansion of the ejecta (Frail et al. 1997a), evidence for a reverse shock (Kulkarni et al. 1999), the first true calorimetry of a GRB explosion (Frail, Waxman \& Kulkarni 1999), the discovery of optically obscured events (Taylor et al. 1998), the first unambiguous evidence that the ejecta are collimated in jets (Harrison et al. 1999), and the discovery of a possible link between supernovae and GRBs (Kulkarni et al. 1998).

\section{RADIO AFTERGLOW STATISTICS}

Since 1997 we have observed 19 GRBs with the VLA and detected a total of eight radio afterglows (see Figure 1, Tables 1 and 2). The peak fluxes $\left(F_{\text {peak }}\right)$ of the detections range from $1200 \mu \mathrm{Jy}$ to $150 \mu \mathrm{Jy}$. This small range of $\mathrm{F}_{\text {peak }}$ values suggests that our ability to detect radio afterglows is severely limited by the sensilivity of the telescope. The "lifetime" (i.e., $t_{\max }$ ) of the radio afterglows is signal-to-noise limited but it is clear, at least among bursts of comparable brightness, that $t_{\max }$ varies substantially. Of special note are the three GRBs $(970828,981226$, and 990506) which have no optical counterparts (i.e., XR class). These may represent an important group of GRBs whose optical cmission is extincted by dust.

There are 11 GRBs for which a VLA search of the error box failed to detect a radio afterglow (see Table 2). The peak fluxes given in the table are conservative upper limits for a radio afterglow on a time-scale of 1 to 30 days and at frequencies betwecn 1.4 and $8.5 \mathrm{GHz}$. These non-detcetions vary in quality depending on the size of the error circle but most observations had sufficient sensitivity to detect radio afterglows with fluxes comparable to those listed in Table 1. 
TABLE 1. Radio Afterglow Detections.

\begin{tabular}{crrrll}
\hline GRB & $\begin{array}{c}F_{\text {peak }} \\
(\mu \mathrm{Jy})\end{array}$ & $\begin{array}{r}\mathrm{t}_{\max } \\
(\text { days })\end{array}$ & $\begin{array}{c}\text { AG } \\
\text { Class }\end{array}$ & Instruments & References \\
\hline 970508 & 1200 & 450 & XOR & VLA, VLBA, OVRO, JCMT & Frail et al. (1997a) \\
970828 & 150 & 3.5 & XR & VLA & Djorgovski et al. (1999) \\
980329 & 300 & 135 & XOR & VLA, OVRO, JCMT & Taylor et al. (1998) \\
$980425^{\mathrm{a}}$ & 50,000 & $>300$ & XOR & ATCA, JCMT & Kulkarni et al. (1998) \\
980519 & 300 & 65 & XOR & VLA, OVRO & Frail et al. (1999a) \\
980703 & 1200 & 210 & XOR & VLA, VLBA, JCMT & Bloom et al. (1998) \\
981226 & 170 & 20 & XR & VLA & Frail et al. (1999b) \\
990123 & 260 & 1.2 & XOR & VLA, OVRO, OVRO 40-m, & \\
& & & & JCMT & Kulkarni et al. (1999) \\
990506 & 550 & $<16$ & XR & VLA & Taylor et al. (1999) \\
990510 & 225 & 20 & XOR & ATCA & Harrison et al. (1999) \\
\hline
\end{tabular}

${ }^{a}$ Related to SN1998bw. We do not include this GRB in the detection statistics.

TABLE 2. VLA Afterglow Non-Detections.

\begin{tabular}{crrl}
\hline GRB & $F_{\text {peak }}(\mu \mathrm{Jy})$ & AG Class & References \\
\hline 970111 & $<300$ & $\mathrm{X}$ & Frail et al. (1997b) \\
970228 & $<50$ & $\mathrm{XO}$ & Frail et al. (1998) \\
970616 & $<150$ & $\mathrm{X}$ & IAUC \#6691 \\
970815 & $<50$ & $\mathrm{X}$ & IAUC \#6723 \\
971214 & $<25$ & $\mathrm{XO}$ & Ramaprakash et al. (1998) \\
971227 & $<50$ & $\mathrm{X}$ & \\
980326 & $<150$ & $\mathrm{O}$ & \\
980613 & $<50$ & $\mathrm{XO}$ & \\
981220 & $<125$ & $\mathrm{X}$ & GCN \#269 \\
990520 & $<125$ & $\mathrm{X}$ & \\
990704 & $<125$ & $\mathrm{X}$ & \\
\hline
\end{tabular}

TABLE 3. ATCA Afterglow Non-Detections.

\begin{tabular}{ccrl}
\hline GRB & $F_{\text {peak }}(\mu \mathrm{Jy})$ & AG Class & References \\
\hline 970402 & $<300$ & $\mathrm{X}$ & \\
980109 & $<550$ & a & \\
990217 & $<175$ & a & GCN \#266 \\
990627 & $<125$ & $\mathrm{X}$ & GCN \#357 \\
990705 & $<100$ & $\mathrm{XO}$ & GCN \#376 \\
990712 & $<100$ & $\mathrm{O}$ & \\
\hline a GRB & 990109 & and 990217 & were seen only in \\
gamma-rays. No afterglows were detected at \\
any wavelength.
\end{tabular}

There have been two radio afterglow detections made at the ATCA (see Table 1). The possible relation of GRB 980425 to SN1998bw makes it a rather unusual event, so we do not include it in the detection statistics. The upper limits of the six ATCA non-detections in Table 3 were not sufficient to have detected the weaker 
radio afterglows in Table 1.

\section{SUMMARY}

In summary, our coordinated program has been very successful in detecting radio afterglows from GRBs. In particular:

- Six gamma-ray bursts are seen at X-ray, optical and radio wavelengths (GRB 970508, GRB 980329, GRB 980519, GRB 980703, GRB 990123, GRB 990510) (see Figure 1).

- Of the $23 \mathrm{X}$-ray afterglows, nine have been detected at radio wavelengths $(\mathrm{XOR}+\mathrm{XR})$ for a rate of $39 \%$. At the VLA the detection rate is $8 / 19$ or $42 \%$. The small range in the observed peak flux densities suggests that our ability to detect radio afterglows is mainly limited by the sensitivity of the telescopes (VLA and ATCA).

- Of the $23 \mathrm{X}$-ray afterglows, ten have been detected at optical wavelengths (XOR + XO) or $43 \%$. The detection rate of well-localized GRBs is comparable at optical and radio wavelengths.

- There exists a growing class (XR) of "dark" GRBs which have X-ray and radio afterglows but no known optical afterglow. These may represent an important group of GRBs whose optical emission is extincted by dust.

\section{REFERENCES}

1. Bloom, J. S., Frail, D. A., Kulkarni, S. R., Djorgovski, S. G., Halpern, J. P., Marzke, R. O., Patton, D. R., Oke, J. B., Horne, K. D., Gomer, R., Goodrich, R., Campbell, R., Moriarty-Schieven, G. H., Redman, R. O., Feldman, P. A., Costa, E., and Masetti, N. The Discovery and Broadband Follow-up of the Transient Afterglow of GRB 980703. ApJ 508, L21-L24, (1998).

2. Djorgovski, S. G., et. al. The radio afterglow from GRB 970828 and optical spectroscopy of its host galaxy. ApJ (Letters) submitted, (1999).

3. Frail, D. A., Kulkarni, S. R., Nicastro, S. R., Feroci, M., and Taylor, G. B. The radio afterglow from the gamma-ray burst of 8 May 1997. Naturc 380, 261 263, (1997a).

4. Frail, D. A., Kulkarni, S. R., Costa, E., Frontera, F., Heise, J., Feroci, M., Piro, L., Dal Fiume, D., Nicastro, L., Palazzi, E., and Jager, R. Radio Monitoring of the 1997 January 11 Gamma-Ray Burst. ApJ 483, L91-L94, (1997b).

5. Frail, D. A., Kulkarni, S. R., Shepherd, D. S., and Waxman, E. No Radio Afterglow from the Gamma-Ray Burst of 1997 February 28. ApJ 502, L119-L122, (1998).

6. Frail, D. A., Kulkarni, S. R., Sari, R., Taylor, G. B., Shepherd, D. S., Bloom, J. S., Young, C. H., Nicastro, L., and Masetti, N. The radio afterglow from GRB 980519: A test of the jet and circumstellar models. ApJ submitted; astro-ph/9910060, (1999a). 
7. Frail, D. A., Kulkarni, S. R., Bloom, J. S., Djorgovski, S. G., Gorjian, V., Gal, R.. R.., Meltzer, J., Sari, R., Chaffee, F. H., Goodrich, R. W., Frontera, F., and Costa, E. The radio afterglow and the host galaxy of the $\mathrm{x}$-ray rich GRB 981226. ApJ (Letters) in press; astro-ph/9909407, (1999b).

8. Frail, D. A., Waxman, E., and Kulkarni, S. R. A 450-day light curve of the radio afterglow of GRB 970508: Fireball calorimetry. ApJ (Letters) submitted; astro$\mathrm{ph} / 9910319,(1999)$.

9. Harrison, F. A., Bloom, J. S., Frail, D. A., Sari, R., Kulkarni, S. R., Djorgovski, S. G., Axelrod, T., Mould, J., Schmidt, B. P., Wieringa, M. H., Wark, R. M., Subrahmanyan, R., McConnell, D., McCarthy, P. J., Schaefer, B. E., McMahon, R. G., Markze, R. O., Firth, E., Soffitta, P., and Amati, L. Optical and Radio Observations of the Afterglow from GRB 990510: Evidence for a Jet. ApJ 523, L121-L124, (1999).

10. Kulkarni, S. R., Frail, D. A., Wieringa, M. H., Ekers, R. D., Sadler, E. M., Wark, R. M., Higdon, J. L., Phinney, E. S., and Bloom, J. S. Radio emission from the unusual supcrnova $1998 \mathrm{bw}$ and its association with the gamma-ray burst of 25 April 1998. Nature 395, 663-669, (1998).

11. Kulkarni, S. R., Frail, D. A., Sari, R., Moriarty-Schieven, G. H., Shepherd, D. S., Udomprasert, P., Readhead, A. C. S., Bloom, J. S., Feroci, M., and Costa, E. Discovery of a Radio Flare from GRB 990123. ApJ 522, L97-L100, (1999).

12. Ramaprakash, A. N., Kulkarni, S. R., Frail, D. A., Koresko, C., Kuchner, M., Goodrich, R., Neugebauer, G., Murphy, R., Eikenberry, S., Bloom, J. S., Djorgovski, S. G., Waxman, E., Frontera, F., Feroci, M., and Nicastro, L. The energetic afterglow of the gamma-ray burst of 14 December 1997. Nature 393, 43-46, (1998).

13. Taylor, G. B. et al. The Discovery of the Radio Afterglow from the Optically Dim Gamma-Ray Burst of 1998 March 29. ApJ 502, L115, (1998).

14. Taylor, G. B. et al. The gamma-ray burst of 1999 May 6 and the population of optically dim afterglows. In preparation, (1999). 